\title{
FATORES ASSOCIADOS À ADAPTAÇÃO ACADÊMICA DE ESTUDANTES DE PSICOLOGIA DO PRIMEIRO PERÍODO
}

\author{
Adriana Benevides Soares ${ }^{1}$ \\ Almir Diego Gonçalves Brito da Silva \\ Bruno de Alcantara Souza \\ Priscila Fernandes Lima \\ Universidade Salgado de Oliveira, Brazil
}

\begin{abstract}
RESUMO
O estudo verificou como as variáveis Expectativas Acadêmicas, Habilidades Sociais, Maturidade para a Escolha Profissional e Educação à Carreira estão associadas à adaptação acadêmica no Ensino Superior no primeiro período do curso de Psicologia. Os participantes foram 214 estudantes de universidades públicas e privadas do Estado do Rio de Janeiro. Utilizaram-se os instrumentos Escala de Expectativas Acadêmicas de Estudantes Ingressantes na Educação Superior, Inventário de Habilidades Sociais, Escala de Maturidade para Escolha Profissional, Questionário de Educação a Carreira e Questionário de Vivências Acadêmicas reduzido. A adaptação mais satisfatória esteve associada a níveis mais realistas de Expectativas Acadêmicas, melhor Educação à Carreira e melhor repertório de Habilidades Sociais. A promoção de competências sociais e de escolha profissional pode contribuir para a adaptação acadêmica no contexto estudado.
\end{abstract}

Palavras-chave

estudantes universitários; curso de Psicologia; adaptação acadêmica; fatores psicossociais; sucesso

\begin{abstract}
This study verified how the variables Academic Expectations, Social Skills, Maturity for Professional Choice and Career Education are associated to the academic adaptation in Higher Education in the first period of the Psychology course. The participants were 214 students from public and private universities in the State of Rio de Janeiro. Scale of Academic Expectations for Higher Education Students, Social Skills Inventory, Maturity Scale for Professional Choice, Career Education Questionnaire and Academic Experience Questionnaire short version were used. The most satisfactory adaptation was associated with more realistic levels of Academic Expectations, better Career Education and better repertoire of Social Skills. The promotion of social skills and professional choice can contribute to the academic adaptation in the studied context.
\end{abstract}

\section{Keywords}

university students; Psychology course; academic adaptation; psychosocial factors; success

1 Correspondence about this article should be addressed to Adrien Benevides Soares.Email: adribenevides@gmail.com 


\section{Factors Associated with Academic Adaptation of Psychology Students in the First Period}

A partir da década de 1990 vê-se ocorrer uma rápida expansão dos cursos de graduação em Psicologia, juntamente a grandes revisões no modelo educacional brasileiro e o oferecimento de programas de acesso ao Ensino Superior, possibilitando o ingresso de jovens provenientes de diferentes classes e perfis sociais (Lisboa \& Barbosa, 2009). Com o ingresso de um maior número de estudantes e com características diversificadas, torna-se relevante compreender as maneiras pelas quais o novo público dos cursos de Psicologia chega à universidade e a este ambiente se adapta, considerando que o processo de transição do Ensino Médio para a Universidade é acompanhado por um conjunto de mudanças que interferem no desenvolvimento psicossocial do jovem (Pinho, Dourado, Aurélio, \& Bastos, 2015).

O fenômeno de adaptação acadêmica é considerado pela literatura como um processo complexo e multidimensional, envolvendo aspectos de natureza intrapessoal e contextual (Soares, Almeida, Diniz, \& Guisande, 2006). A qualidade das experiências durante o primeiro ano na universidade interfere na permanência e no sucesso acadêmico dos alunos, de modo que estudantes mais integrados, acadêmica e socialmente, tendem a alcançar maior êxito intelectual e pessoal do que aqueles que vivenciam dificuldades no período de transição à universidade (Teixeira, Dias, Wottrich, \& Oliveira, 2008). As principais dificuldades na adaptação estão ligadas a problemas oriundos das exigências acadêmicas, dos desafios nas relações interpessoais, das mudanças na formação da identidade e do desenvolvimento vocacional dos jovens. Fatores relacionados à instituição de ensino têm também relevância neste processo. A infraestrutura; os serviços oferecidos; a organização curricular; as atividades pedagógicas e as extracurriculares; as políticas educacionais e a competência dos docentes nos âmbitos pedagógicos, científicos e interpessoais devem ser considerados na compreensão do sucesso escolar do estudante universitário (Brites-Ferreira, Seco, Canastra, Simões-Dias, \& Abreu, 2011).

Soares, Almeida, Diniz e Guisande (2006) contribuíram para a compreensão do processo de adaptação acadêmica procurando considerar as formas de alguns jovens lidarem com as exigências da vida universitária. No Modelo Multidimensional de Ajustamento de jovens ao contexto Universitário (MMAU) foram consideradas as características individuais dos estudantes, como informações sociodemográficas, acadêmicas e desenvolvimentais e os fatores relacionados à qualidade das universidades, como a infraestrutura, os recursos e os serviços. Compreende-se, portanto, que a experiência de ajustamento ao contexto universitário está ligada a fatores pessoais e contextuais que, relacionados, podem oferecer uma compreensão mais satisfatória deste fenômeno.

Com o objetivo de verificar o papel do ambiente universitário na transição de estudantes ao Ensino Superior e verificar o perfil destes alunos, Sarriera, Paradiso, Schütz e Howes (2012) realizaram uma pesquisa com 273 estudantes do curso de Psicologia. A amostra contou com $84 \%$ de mulheres e $67,4 \%$ de alunos provenientes do Ensino Médio cursado em rede privada. Os resultados mostraram que os estudantes se encontravam medianamente adaptados. Um dos aspectos mais relevantes foi a forma como os alunos perceberam-se envolvidos com o curso e a profissão escolhida, acreditando que conseguirão, no futuro, concretizar suas expectativas em relação aos seus anseios vocacionais. Os estudantes se diferenciaram quanto às questões pertinentes às dimensões pessoais, de estudo e institucionais, corroborando a ideia de que as características pessoais e da instituição estão associadas ao processo de adaptação acadêmica.

As expectativas acadêmicas são uma das variáveis mais estudadas no contexto da adaptação a Universidade conforme Fernandes e Almeida (2005) e podem ser definidas como sendo predições que a pessoa faz em relação ao seu desempenho em um determinado contexto social para atender não só as suas necessidades mas, também as do outro (Gomes \& Soares, 2013). Segundo Marinho-Araújo, Fleith, Almeida, Bisinoto e Rabelo (2015), as expectativas são fundamentais na transição do Ensino Médio para o Superior e na permanência dos estudantes no curso escolhido na medida em que influenciam a forma como enfrentam vários desafios dos quais não esperavam em suas projeções (Moreno \& Soares, 2014). Nesse sentido, parecem afetar os níveis de compromisso e de investimento dos alunos, criando uma instabilidade na adaptação acadêmica (Marinho-Araújo et al., 2015). Diversos estudos têm mostrado esta relação (Gomes \& Soares, 2013; Nadelson et al., 2013; Oliveira, Santos, \& Dias, 2016; Soares et al., 
2014). O estudo de Nadelson et al. (2013) preocupou-se em verificar possíveis fatores que pudessem influenciar a escolha de 351 estudantes americanos ingressantes e a relação de suas expectativas e experiências com a universidade. Em termos de influências percebidas pelos alunos sobre suas decisões de frequentar um curso superior, constatou-se que são feitas por razões pessoais como "carreira" e "aprender coisas que me interessam". A pesquisa apontou que campanhas publicitárias e competições esportivas universitárias, podem influenciar na decisão dos discentes no processo de entrada à faculdade. Constatouse que, em geral, que as experiências dos alunos foram satisfatórias pois, a infraestrutura que a instituição disponibiliza parece favorecer o desenvolvimento dos estudantes. Por outro lado, as percepções quanto aos professores exclusivos para o público iniciante e o foco nas pesquisas da universidade não foram positivas.

No cenário nacional, Oliveira, Santos e Dias (2016) entrevistaram 20 estudantes, entre calouros e formandos, dos cursos de Economia e de Psicologia de uma universidade pública no interior do estado do Rio Grande do Sul. Constataram que os acadêmicos esperavam mais integração com os outros colegas, melhor relacionamento com os professores, mais informações sobre a universidade e o curso escolhido. Além disso, esperavam salas de aula mais equipadas, laboratórios de informática, prédio com melhor infraestrutura. Dessa forma, os autores mostram que a estrutura da universidade pode influenciar no processo de adaptação acadêmica dos alunos. Um outro estudo de Soares et al. (2014) com 182 estudantes de universidades públicas e privadas do estado do Rio de Janeiro de diversos cursos buscou analisar as relações entre as expectativas e as vivências acadêmicas de estudantes ingressantes. Os resultados apontaram que as expectativas de envolvimento social, projeto vocacional de carreira e atividades curriculares são preditores da qualidade das vivências acadêmicas. Nesta mesma direção, a pesquisa realizada por Gomes e Soares (2013) verificou a existência de correlações entre expectativas acadêmicas, habilidades sociais (HS) e inteligência em 196 alunos no primeiro ano de universidades públicas e privadas, dos cursos da área de ciências humanas, saúde e educação. A pesquisa apontou que alunos com expectativas menos realistas em relação à instituição e os recursos de infraestrutura têm desempenho inferior àqueles com expectativas mais realistas que por sua vez apresentam mais envolvimento curricular e vocacional. Para os pesquisadores, os alunos ingressantes que tem mais autonomia e exploram mais seus recursos pessoais têm expectativas mais realistas em relação ao que vão encontrar como infraestrutura institucional. $\mathrm{O}$ estudo também evidenciou a correlação entre expectativas acadêmicas e HS em que estudantes que apresentam maiores escores de HS têm mais expectativas na área vocacional, curricular e social.

Além das expectativas, outros estudos têm mostrado uma forte relação entre o ajustamento acadêmico e a competência social dos estudantes (A. Del Prette \& Del Prette, 2003). Os autores afirmam que a adesão ao curso de nível superior não está unicamente vinculada a competências técnicas e instrumentais mas, também a aspectos atrelados às relações interpessoais desenvolvidas neste contexto.

Pessoas socialmente competentes estabelecem relações interpessoais mais produtivas, maior satisfação e motivação pessoal, pois apresentam melhor saúde mental e física quando comparadas àquelas que possuem déficits em habilidades sociais (Colombo \& Prati, 2014). É possível definir o termo HS como um conjunto de comportamentos expressados no repertório do indivíduo diante das situações interpessoais (A. Del Prette \& Del Prette, 2013). Observa-se neste sentido, uma demanda do mercado de trabalho para que as instituições de Ensino Superior busquem conjugar a competência técnica à social fazendo com que o estudante possa estar mais preparado para atuar profissionalmente (A. Del Prette \& Del Prette, 2003).

Na realidade, a adaptação acadêmica requer não só recursos cognitivos e afetivos mas, em grande parte, também recursos sociais que possibilitem o sucesso do aluno ao longo do percurso universitário (Nelson, Quinn, Marrington, \& Clarke, 2012). As habilidades sociais podem contribuir para o aproveitamento cognitivo e escolar, uma melhor regulação da afetividade e um maior autocontrole da agressividade, além de uma forma mais madura e assertiva de relacionar-se com os colegas e professores (Soares \& Del Prette, 2015). Visando o mesmo objetivo, de relacionar HS com o ajustamento do estudante à universidade, alguns estudos mostraram resultados empíricos entre estas variáveis (Pellegrini, Calais, \& Salgado, 2012; Soares, Seabra, \& Gomes, 2014).

ARTICLES $\mid 433$ 
Pellegrini et al. (2012) estudaram a relação entre as variáveis HS, administração do tempo e estresse em 83 calouros. Constatou-se que a incidência de estresse, o déficit em habilidades sociais e a inadequada administração do tempo comprometem a saúde dos universitários, já que o estresse implica em grande gasto de energia e desgaste físico. A relação entre habilidades sociais e administração do tempo e desta última com o estresse apontou uma associação indireta entre deficiências em HS e presença de estresse.

Soares, Seabra e Gomes (2014) identificaram correlações entre autoeficácia acadêmica, inteligência e HS de 162 universitários. Em relação ao gênero, foram encontradas diferenças significativas, sendo as mulheres mais competentes socialmente com escores maiores em Conversação e Desenvoltura Social e os homens, mais capazes cognitivamente. Os estudantes de instituições públicas obtiveram escores superiores aos das privadas. Sendo assim, foi reforçada a necessidade da presença de habilidades cognitivas e comportamentais como promotoras do bem-estar psicológico e físico dos universitários.

O momento de transição à vida adulta exige do jovem não só competências interpessoais para a realização de tarefas, mas também um nível de maturidade para a escolha profissional. O ideal é que ao longo do desenvolvimento, o indivíduo aprimore o seu repertório de comportamentos interpessoais de acordo com a demanda do meio social em que vive estabelecendo círculos de amizades, participação em atividades de seu interesse dentre outras HS e psicológicas tornando-se mais autônomo (Colombo \& Prati, 2014).

Definir a carreira depende, em grande parte, do conhecimento que o indivíduo possui sobre as opções profissionais existentes em sua realidade e do autoconhecimento, ou seja, a identificação dos seus interesses, valores, habilidades e motivações. Considerada um processo multifatorial que sofre interferência dos aspectos políticos, econômicos, sociais, educacionais, familiares e psicológicos, pode-se conceber a escolha profissional como um período de transição com possibilidades desestabilizadoras (Sparta \& Gomes, 2005). Entende-se por maturidade para a escolha profissional, o conjunto de atitudes e conhecimentos que o indivíduo acumula e que o prepara para a tomada de decisão consciente, consistindo em comportamentos que devem ser empreendidos na inserção no mundo do trabalho (Neiva, 2014). Tornar-se adulto, requer competências para a tomada de decisões que proporcionam ao jovem a capacidade de conduzir sua própria vida (Junqueira \& Melo-Silva, 2014).

Com o objetivo de verificar os aspectos determinantes apresentados no processo de escolha profissional, estudos na área têm sido cada vez mais frequentes. No cenário internacional, Ulusoy e Onen (2014) analisaram os efeitos dos níveis de maturidade profissional em 607 estudantes do Ensino Médio de Ancara, Turquia. Os resultados mostraram que os alunos com baixos níveis de maturidade profissional tinham baixa motivação acadêmica, enquanto estudantes com altos níveis tinham motivações acadêmicas elevadas. No Brasil, o estudo de Neiva, Silva, Miranda e Esteves (2005) identificou diferenças significativas na maturidade para a escolha profissional de 950 estudantes do Ensino Médio. As moças mostraram-se mais maduras do que os rapazes, os alunos de escola particular mais maduros do que os da pública e os do terceiro ano, mais do que os do primeiro.

Durante o Ensino Médio espera-se que o adolescente já tenha alguma maturidade para escolher sua carreira. No entanto, sabe-se que esse processo de tomada de decisão envolve múltiplos fatores que podem comprometer uma escolha mais consciente da sua futura profissão. É importante que o jovem desenvolva determinadas competências e que tenha algum tipo de orientação, de modo que consiga decidir com mais qualidade seu rumo profissional.

O desenvolvimento de carreira tem se destacado em diversos estudos na área da psicologia vocacional. Blustein (2011) ressalta a importância das relações na vida das pessoas e o apoio de suas referências neste processo. $\mathrm{O}$ autor acredita no impacto de outras pessoas como família, amigos, psicólogos e professores no processo de desenvolvimento de carreira do sujeito em detrimento da elaboração de projetos profissionais. Apesar de alguns estudos incidirem no rendimento acadêmico, a importância do papel dos professores na educação à carreira dos alunos também tem se destacado em nível nacional e internacional (Gamboa \& Paixão, 2014; Oliveira, Taveira, \& Neves, 2014). 
No Brasil, os estudos na área ainda são iniciantes quando comparados a outras pesquisas na área da orientação profissional (Uvaldo, Garcia, Munhoz, \& Teixeira, 2012). O termo educação à carreira tem sido pesquisando por autores como Carvalho e Marinho-Araújo (2010), que afirmaram que a implantação da orientação profissional na escola por meio de uma política pública, seria um avanço não só por favorecer a educação à carreira mas, também pela necessidade do psicólogo escolar.

Nesse sentido, pesquisas como a de Faleiros e Lehman (2016) e Teixeira e Calado (2010), se dispuseram a olhar de forma mais profunda a realidade escolar. Faleiros e Lehman (2016) evidenciaram o resultado da proposta de Educação para a Carreira desenvolvida em alunos do $1^{\circ}$ ano do Ensino Médio de uma escola técnica paulista nos anos de 2012 e 2013 por meio das aulas que foram integradas à carga horária regular dos alunos, constatou que as aulas proporcionaram um importante espaço de reflexão para os alunos a respeito do mundo do trabalho. O estudo de Teixeira e Calado (2010) propôs um programa de intervenção com a perspectiva de desenvolvimento da profissão e da construção da cidadania fundamentada nos princípios da Educação à Carreira. Participaram da pesquisa 53 alunos do $9^{\circ}$ ano do ensino regular de duas escolas do Alentejo, Portugal. Após a intervenção, ocorreram mudanças nos domínios de desenvolvimento pessoal como autoconhecimento, planejamento pessoal, informação sobre formação e opções profissionais. Por outro lado, os itens cujas diferenças não apresentam significância estatística apontaram questões relacionadas à pressão dos colegas nas escolhas, às oportunidades profissionais e às probabilidades de emprego, além da associação entre as atividades de tempos livres e os objetivos profissionais que permite conjugar recursos e potencializar o desenvolvimento das pessoas e das instituições.

Uma preparação adequada no período pré-universitário favorece o estudante a desenvolver determinadas competências que auxiliam no processo de adaptação acadêmica. Estudantes que se preparam ou recebem algum tipo de orientação vocacional são mais propensos a permanecer no curso escolhido (Balbinotti \& Tétrau, 2006). A escolha profissional madura exige que o jovem possua um conjunto de comportamentos e atitudes como determinação, responsabilidade e independência (Neiva, 2003). Características pessoais como a maturidade para a escolha profissional contribuem para uma melhor adaptação acadêmica. Além disso, um bom repertório de habilidades sociais pode proporcionar vivências mais saudáveis e ainda favorecer o rendimento, o que facilitaria o processo de transição para o Ensino Superior (Soares \& Del Prette, 2015). Assim, estudantes que possuem projetos vocacionais mais definidos, desejo de participação nas atividades acadêmicas e expectativas de envolvimento com a instituição, com o curso e nas relações com os colegas tendem a realizar um ajustamento mais positivo (Soares et al., 2014).

Pelo fato de ser um processo multidimensional, a adaptação acadêmica carece de uma investigação que considere os fatores pessoais dos participantes, assim como os aspectos institucionais e culturais. Desse modo, realizar uma pesquisa focada em determinado curso é positivo no que diz respeito ao conhecimento mais aprofundado das realidades inerentes ao seu contexto. Refletir sobre aspectos relativos ao sucesso durante o curso de Psicologia pode contribuir na construção das práticas de ensino nesta profissão e auxiliar a alunos de diferentes condições sociais a conseguir integrar-se de forma satisfatória nesta modalidade de ensino. Tendo em vista a literatura apresentada, este estudo tem por objetivo verificar a relação preditiva entre as variáveis explicativas Expectativas Acadêmicas, Habilidades Sociais, Maturidade para a Escolha Profissional, Educação à Carreira e a variável critério Adaptação à Universidade em estudantes universitários do primeiro período de Psicologia.

\section{Método}

\section{Participantes}

Participaram, escolhidos por conveniência, 214 estudantes universitários do primeiro período do curso de Psicologia de universidades públicas $(22,0 \%)$ e privadas $(78,0 \%)$ do Estado do Rio de Janeiro com idades variando entre 17 e 52 anos $(M=24,04 ; D P=7,93)$, sendo $46(21,5 \%)$ homens e $168(78,5 \%)$ mulheres. Dentre eles, $173(80,8 \%)$ eram solteiros; $31(14,5 \%)$ casados e $10(4,7 \%)$ declararam outros 
estados civis. Pertenciam $14(6,5 \%)$ a classe social A; $10(4,7 \%)$ a B1; $77(36,0 \%)$ a B2; $67(31,3 \%)$ a $\mathrm{C} 1$; $36(16,8 \%)$ a $\mathrm{C} 2$ e $10(4,7 \%)$ a $\mathrm{D} / \mathrm{E}$.

\section{Procedimentos}

A aplicação dos instrumentos foi realizada de forma coletiva, em horário de aula. Foram aplicados os seis instrumentos sem restrição de tempo. Os dados sociodemográficos foram coletados em folha de rosto que se precedeu à aplicação dos instrumentos. O projeto foi encaminhado e aprovado pelo Comitê de Ética em Pesquisa da Universidade. Todos os participantes assinaram o Termo de Consentimento Livre e Esclarecido. Foi realizada Regressão Linear Múltipla considerando como variáveis explicativas Expectativas Acadêmicas, Habilidades Sociais, Maturidade para a Escolha Profissional, Educação à Carreira e a variável critério Adaptação à Universidade.

\section{Instrumentos}

Escala de Expectativas Acadêmicas de Estudantes Ingressantes na Educação Superior (EEAEIES) (Almeida, Costa, Alves, Gonçalves, \& Araújo, 2012; adaptado por Marinho-Araújo et al., 2015). É um instrumento composto por 61 itens e sete fatores: 1) Formação Acadêmica de Qualidade (12 itens e $\alpha=0,82)$; 2) Compromisso Social e Acadêmico (13 itens e $\alpha=0,90)$; 3) Ampliação das Relações Interpessoais (oito itens e $\alpha=0,84$ ); 4) Oportunidade de Internacionalização e Intercâmbio (oito itens e $\alpha=0,87$ ); 5) Perspectiva de Sucesso Profissional (cinco itens e $\alpha=0,71$ ); 6) Preocupação com a Autoimagem (seis itens e $\alpha=0,78$ ) e 7) Desenvolvimento de Competências Transversais (nove itens e $\alpha=0,79)$.

Inventário de Habilidades Sociais (IHS) (Z. A. P. Del Prette, \& A. Del Prette, 2001). É um instrumento de autorrelato (38 itens) e cinco fatores: 1) Enfrentamento e Autoafirmação com Risco (11 itens e $\alpha=0,96)$; 2) Autoafirmação na Expressão de Sentimento Positivo (sete itens e $\alpha=0,86$ ); 3) Conversação e Desenvoltura Social (sete itens e $\alpha=0,81$ ); 4) Autoexposição a Desconhecidos e Situações Novas (quatro itens e $\alpha=0,75$ ); 5) Autocontrole da Agressividade (três itens e $\alpha=0,74$ ).

Escala de Maturidade para Escolha Profissional (EMEP) (Neiva, 2014). É composta por 23 itens positivos (que indicam maturidade) e 22 negativos (que indicam imaturidade) e cinco subescalas: 1) Determinação (10 itens e $\alpha=0,91)$; 2) Responsabilidade (10 itens e $\alpha=0,75$ ); 3) Independência (oito itens e $\alpha=0,75)$; 4) Autoconhecimento (sete itens e $\alpha=0,75)$ e 5) Conhecimento da Realidade Profissional (10 itens e $\alpha=0,82$ ).

Questionário de Educação à Carreira (QEC) (Dupont \& Gingras, 1990, adaptado por Balbinotti \& Tétreaux, 2006). É um instrumento de 96 itenscomposto por duas subescalas e seis dimensões relativas a segunda subescala: 1) Sentido e Importância do Trabalho (22 itens e $\alpha=0,81) ; 2$ ) Preparação à Carreira: 2.1) Passos Efetuados (13 itens e $\alpha=0,78)$; 2.2) Fatores Considerados (17 itens e $\alpha=0,84) ; 2.3$ ) Profissão Preferida (nove itens e $\alpha=0,87) ; 2.4)$ Pesquisa e Conservação de Emprego (11 itens e $\alpha=0,89 ; 2.5$ ) Pessoas e Fontes Consultadas (11 itens e $\alpha=0,73$ ) e 2.6) Atividades Realizadas (13 itens e $\alpha=0,84$ ).

Questionário de Vivências Acadêmicas - versão reduzida (QVA-r) (Almeida, Soares, \& Ferreira, 2002, adaptado por Granado, Santos, Almeida, Soares, \& Guisande, 2005). É um instrumento de autorrelato composto de 55 itens estão divididos em cinco dimensões: 1) Pessoal (14 itens e $\alpha=0,84$ ); 2) Curso-carreira (12 itens e $\alpha=0,86$ ); 3) Estudo (nove itens e $\alpha=0,78)$; 4) Interpessoal (12 itens e $\alpha=$ 0,82 ); 5) Institucional (oito itens e $\alpha=0,77$ ).

\section{Resultados}

Os resultados da Tabela 1 apontam que o fator "Ampliação das Relações Interpessoais" da escala de EEAEIES e o escore total de HS apresentaram impacto na dimensão Pessoal das Vivências Acadêmicas, explicando $28 \%$ e $20 \%$ das vivências pessoais respectivamente. Os fatores da EMEP, "Responsabilidade" e "Autoconhecimento" e seu escore total apresentaram impacto negativo, explicando $22 \%, 21 \%$ e $35 \%$ do fenômeno, respectivamente. 
Tabela 1

Análise de regressão considerando como VD Vivência Acadêmica Pessoal

\begin{tabular}{lllcc}
\hline & & $\mathrm{R}^{2}=0,23$ & $\mathrm{~F}=3,07$ & $\mathrm{p}<0,001$ \\
\hline $\begin{array}{l}\text { Variáveis } \\
\text { Independentes }\end{array}$ & Fatores & $\mathbf{B}$ & $\boldsymbol{t}$ & $\boldsymbol{p}$ \\
\hline Expectat. Acad. & Amp. Rel. Int. & 0,28 & 2,54 & $0,01^{*}$ \\
Maturidade & Responsabilidade & $-0,22$ & $-2,33$ & $0,02^{*}$ \\
& Autoconhecimento & $-0,21$ & $-2,20$ & $0,03^{*}$ \\
\hline & & $\mathrm{R}^{2}=0,15$ & $\mathrm{~F}=8,24$ & $\mathrm{p}<0,001$ \\
\hline Habilid. Sociais & & 0,20 & 2,99 & $0,01^{*}$ \\
Maturidade & & $-0,35$ & $-5,14$ & $0,00^{*}$ \\
\hline
\end{tabular}

$* p<0,05$

Foi encontrado, conforme a Tabela 2, que os escores totais das variáveis independentes "Expectativas Acadêmicas" e "Educação à Carreira" são preditores positivos da variável Vivência Acadêmica Estudo explicando $23 \%$ e $28 \%$ do fenômeno, respectivamente. Também o fator "Pessoas e Fontes Consultadas" do QEC obteve impacto positivo explicando 19\% na variável dependente.

Tabela 2

Análise de regressão considerando como VD Vivência Acadêmica Estudo

\begin{tabular}{|c|c|c|c|c|}
\hline ( & (2) & $\mathbf{R}^{2}=0,27$ & $F=\mathbf{3 , 5 2}$ & $\mathrm{p}<\mathbf{0 , 0 0 1}$ \\
\hline $\begin{array}{l}\text { Variáveis } \\
\text { Independentes }\end{array}$ & Fatores & B & $t$ & $p$ \\
\hline \multirow[t]{2}{*}{ Educ. à Carreira } & $\begin{array}{l}\text { Pessoas e fontes } \\
\text { consultadas }\end{array}$ & 0,19 & 2,17 & $0,03 *$ \\
\hline & & $\mathrm{R}^{2}=0,26$ & $F=15,59$ & $\mathrm{p}<0,001$ \\
\hline Exp. Acadêmica & & 0,23 & 3,37 & $0,01 *$ \\
\hline Educ. à Carreira & & 0,28 & 3,83 & $0,00^{*}$ \\
\hline
\end{tabular}

$* p<0,05$

A dimensão Interpessoal da variável Vivência Acadêmica foi impactada positivamente pelos escores totais da EEAEIES, IHS e QEC, explicando 25\%, 22\% e 36\% da variável dependente, conforme apresentado na Tabela 3. Foram identificadas também relações preditivas dos fatores "Compromisso Social e Acadêmico" e "Ampliação das Relações Interpessoais" da EEAEIES, "Fatores Considerados" e "Pessoas e Fontes Consultadas" do QEC, explicando, respectivamente, 23\%, 24\%, 19\% e 23\% da variável dependente. 
Tabela 3

Análise de regressão considerando como VD Vivência Acadêmica Interpessoal

\begin{tabular}{llccc}
\hline & & $\mathbf{R}^{\mathbf{2}}=\mathbf{0 , 3 6}$ & $\mathbf{F}=\mathbf{4 , 9 1}$ & $\mathbf{p}<\mathbf{0 , 0 0 1}$ \\
\hline $\begin{array}{l}\text { Variáveis } \\
\text { Independentes }\end{array}$ & Fatores & $\mathbf{B}$ & $\boldsymbol{t}$ & $\boldsymbol{p}$ \\
\hline Expectat. Acad. & Comp. Soc. e Acad. & 0,23 & 2,02 & $0,04^{*}$ \\
& Amp. Rel. Int. & 0,24 & 2,40 & $0,02^{*}$ \\
Educ. à Carreira & Fatores considerados & 0,19 & 2,01 & $0,04^{*}$ \\
& Pessoas e fontes & 0,23 & 2,80 & $0,01^{*}$ \\
& consultadas & $\mathrm{R}^{2}=0,29$ & $\mathrm{~F}=17,86$ & $\mathrm{p}<0,001$ \\
\hline & & 0,25 & 3,68 & $0,00^{*}$ \\
Exp. Acadêmica & & 0,22 & 3,60 & $0,00^{*}$ \\
Habilid. Sociais & & 0,36 & 5,01 & $0,00^{*}$ \\
Educ. à Carreira & & &
\end{tabular}

$* p<0,05$

De acordo com a Tabela 4, foi encontrado que as variáveis independentes Expectativas Acadêmicas e Educação à Carreira são preditoras da Vivência Acadêmica Institucional, explicando 18\% e $22 \%$, assim como os fatores "Oportunidade de Internacionalização e Intercâmbio" da EEAEIES, "Pessoas e Fontes Consultadas" do QEC e "Determinação" da EMEP que explicam, respectivamente, 33\%, 28\% e $25 \%$ do fenômeno. Foi encontrado impacto negativo para os fatores "Desenvolvimento de Competências Transversais" da EEAEIES, "Enfrentamento e Autoafirmação com Risco" do IHS e "Conhecimento da Realidade Profissional" da EMEP explicando, respectivamente, $42 \%, 19 \%$ e $17 \%$ da variável dependente.

Tabela 4

Análise de regressão considerando como VD Vivência Acadêmica Institucional

\begin{tabular}{llccc}
\hline & & $\mathbf{R}^{\mathbf{2}=\mathbf{0 , 2 2}}$ & $\mathbf{F}=\mathbf{2 , 9 3}$ & $\mathbf{p}<\mathbf{0 , 0 0}$ \\
\hline $\begin{array}{l}\text { Variáveis } \\
\text { independentes }\end{array}$ & Fatores & $\mathbf{B}$ & $\boldsymbol{t}$ & $\boldsymbol{p}$ \\
\hline Expectat. Acad. & Opor. Int. e Int. & 0,33 & 2,94 & $0,01^{*}$ \\
& Des. Comp. Trans & $-0,42$ & $-3,56$ & $0,00^{*}$ \\
Habilid. Sociais & Enfrentamento & $-0,19$ & $-1,99$ & $0,04^{*}$ \\
Educ. à Carreira & Pessoas e fontes consultadas & 0,28 & 2,99 & $0,01^{*}$ \\
Maturidade & Determinação & 0,25 & 2,42 & $0,02^{*}$ \\
& Conhec. da real prof & $-0,17$ & $-2,06$ & $0,04^{*}$ \\
\hline & & $\mathrm{R}^{2}=0,08$ & $\mathrm{~F}=4,58$ & $\mathrm{p}<0,01$ \\
\hline Exp. Acadêmica & & 0,18 & 2,340 & $0,02^{*}$ \\
Educ. à Carreira & & 0,22 & 2,629 & $0,01^{*}$ \\
\hline
\end{tabular}

$* p<0,05$

As Expectativas Acadêmicas e a Maturidade para a Escolha Profissional mostraram impacto positivo na Vivência Acadêmica Carreira assim como também o fator "Compromisso Social e Acadêmico" da EEAEIES, explicando, respectivamente, 16\%, 16\% e 30\% da variável dependente, como apresentado na Tabela 5. 
Tabela 5

Análise de regressão considerando como VD Vivência Acadêmica Carreira

\begin{tabular}{|c|c|c|c|c|c|}
\hline 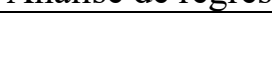 & (2) & & $R^{2}=0,24$ & $F=3,15$ & $p<0,001$ \\
\hline $\begin{array}{l}\text { Variáveis } \\
\text { Independentes }\end{array}$ & Fatores & & B & $t$ & $p$ \\
\hline \multirow[t]{2}{*}{ Expectat. Acad. } & $\begin{array}{l}\text { Comp. Soc. } \\
\text { Acad. }\end{array}$ & $\mathrm{e}$ & 0,30 & 2,46 & $0,02 *$ \\
\hline & & & $\mathrm{R}^{2}=0,19$ & $\mathrm{~F}=10,48$ & $\mathrm{p}<0,001$ \\
\hline Exp. Acadêmica & & & 0,16 & 2,23 & $0,03 *$ \\
\hline Maturidade & & & 0,16 & 2,37 & $0,02 *$ \\
\hline
\end{tabular}

$* p<0,05$

Por fim, na Tabela 6, o fator "Pessoas e Fontes Consultadas" do QEC apresentou impacto positivo no QVA-r assim como os escores totais das variáveis Expectativas Acadêmicas, Habilidades Sociais e Educação à Carreira, explicando 26\%,23\%,21\% e 35\% do fenômeno, respectivamente.

Tabela 6

Análise de regressão considerando como VD Vivência Acadêmica total

\begin{tabular}{llccc}
\hline & & $\mathbf{R}^{\mathbf{2}=\mathbf{0 , 2 9}}$ & $\mathbf{F}=\mathbf{3 , 7 9}$ & $\mathbf{p}<\mathbf{0 , 0 0 1}$ \\
\hline $\begin{array}{l}\text { Variáveis } \\
\text { independentes }\end{array}$ & Fatores & $\mathbf{B}$ & $\boldsymbol{t}$ & $\boldsymbol{P}$ \\
\hline Educ. à Carreira & Pessoas e fontes consultadas & 0,26 & 2,91 & $0,01^{*}$ \\
\hline & & $\mathrm{R}^{2}=0,26$ & $\mathrm{~F}=15,06$ & $\mathrm{p}<0,001$ \\
\hline Exp. Acadêmica & 0,23 & 3,38 & $0,01^{*}$ \\
Habilid. Sociais & 0,21 & 3,31 & $0,01^{*}$ \\
Educ. à Carreira & 0,35 & 4,72 & $0,00^{*}$ \\
\hline
\end{tabular}

$* p<0,05$

Discussão

As associações negativas dos fatores Responsabilidade e Autoconhecimento da EMEP e do seu escore total com a Vivência Acadêmica Pessoal apontam que quanto mais os estudantes se preocupam e se empenham na busca de informações que possam contribuir para sua escolha profissional, tendem a experimentar mal-estar e angústia psicológica podendo inclusive criar uma instabilidade para o seu desenvolvimento acadêmico. Resultados similares foram encontrados no estudo de Soares, Poubel e Mello (2009) que afirma que alunos que apresentam um grau elevado de compromisso com suas rotinas acadêmicas e buscam as melhores opções para se inserirem no mundo do trabalho, podem passar por situações desafiadoras e difíceis levando-os a desconfortos emocionais e físicos.

Ao contrário destes aspectos que podem se configurar como estressores, as relações interpessoais têm um papel relevante na busca do bem-estar durante a adaptação à vida universitária como demonstrado pelas associações positivas entre o escore total do IHS, com a Vivência Acadêmica Pessoal e os fatores Ampliação das Relações Interpessoais do EEAEIES. De fato, estudantes que possuem relacionamentos saudáveis tendem a obter uma melhor realização pessoal (Teixeira, Dias, Wottrich, \& Oliveira, 2008) e indivíduos que possuem um repertório mais elaborado de HS estabelecem melhores relações interpessoais e têm maior satisfação pessoal, além de apresentarem boa saúde física e mental (Colombo \& Prati, 2014). Ainda no âmbito interpessoal, foi verificada a associação positiva da adaptação nesta vivência acadêmica com o escore total da EEAEIES e seus fatores Compromisso Social e Acadêmico, Ampliação das Relações, com as Habilidades Sociais e com a Educação à Carreira e suas subescalas Fatores 
Considerados e Pessoas e Fontes consultados. Estas relações corroboram a pesquisa de Oliveira, Santos e Dias (2016) quando afirma que os estudantes esperam estabelecer uma integração com os colegas de curso e com os professores e demonstram ainda que as relações interpessoais podem ser uma importante fonte de orientação no período pré-universitário. Além disso, um bom repertório de HS em seu fator Enfrentamento e Autoafirmação com Risco pode estar ligado a uma maior capacidade em lidar com diferentes interlocutores no contexto acadêmico, principalmente com figuras de autoridade e funcionários do campus universitário. De fato, as pesquisas feitas por Nelson et al. (2012) também indicam que o processo adaptativo acadêmico requer, em grande parte, de outros recursos sociais como cognitivos e afetivos que possam possibilitar o sucesso do discente ao longo da sua trajetória no Ensino Superior.

Assim como as realidades ligadas às relações interpessoais, a Educação para a Carreira relacionou-se positivamente à adaptação acadêmica, sendo associada aos fatores Estudo, Interpessoal e Institucional do QVA-r. Corroborando assim a necessidade do oferecimento desta modalidade educacional durante a Educação Básica como apontado por Balbinotti e Tétreau (2006). Estudantes que se preparam ou recebem algum tipo de orientação vocacional são mais propensos a permanecerem no curso escolhido. A orientação vocacional destinada aos alunos antes do ingresso à universidade colabora para o desenvolvimento de determinadas competências que o auxiliarão no processo de adaptação acadêmica evidenciando que a escola tem um importante papel na vida profissional do aluno ao dar o suporte à carreira (Figueiredo, Filho, \& Santos, 2015). Pode-se perceber ainda, que quanto maior a interação social nas instituições em que estudam maior a possibilidade de satisfação interpessoal (Guichard, 2001).

Dificuldades em relação ao estudo e ao desempenho acadêmico contribuem para a evasão escolar. A Vivência Acadêmica Estudo foi associada de forma positiva às Expectativas Acadêmicas, à Educação à Carreira e ao seu fator Pessoas e Fontes Consultadas. Desta forma, vê-se que alunos mais capazes de gerenciar suas atividades acadêmicas são aqueles que apresentaram maiores expectativas acadêmicas, ou seja, pensamentos, motivações ou afetos em relação às experiências universitárias (Soares et al., 2014) que podem contribuir para um maior compromisso ou esforço com as tarefas.

Assim como a Educação à Carreira, a Maturidade para Escolha Profissional está associada positivamente à Vivência Acadêmica Institucional e Carreira por meio do fator Determinação e de seu escore total, respectivamente. Demonstrando assim a satisfação do estudante com a escolha pelo curso escolhido e apresentando a Determinação, não somente associada ao curso escolhido, mas também à instituição frequentada, o que poderia contribuir para o estudante suportar as dificuldades encontradas no local de estudo e apresentando maiores níveis de satisfação com a Universidade ou com seu campus acadêmico.

Em todos os seus fatores, as Vivências Acadêmicas foram associadas positivamente às Expectativas. Estes dados corroboram a pesquisa de Marinho-Araújo et al. (2015). As expectativas acadêmicas parecem afetar os níveis de compromisso e de investimento dos estudantes em relação ao curso escolhido impactando, dessa forma, a maneira que se integram ao ambiente universitário. Um outro estudo, realizado por Soares et al. (2014), aponta que as expectativas no que corresponde ao envolvimento social, projeto vocacional de carreira e atividades curriculares são preditoras da qualidade das vivências acadêmicas.

\section{Considerações finais}

O presente estudo contribui para a compreensão do fenômeno de adaptação acadêmica ao relacioná-lo com fatores psicossociais inerentes à preparação para a vida universitária e ao período de ingresso. Pelo seu caráter multidimensional, as vivências acadêmicas puderam ser verificadas em seus diversos fatores através das correlações com as variáveis Expectativas Acadêmicas, Habilidades Sociais, Maturidade para a Escolha Profissional e Educação à Carreira. Aprofundar o conhecimento sobre a experiência inicial dos estudantes universitários pode ser um caminho para a melhoria do processo educacional, com implantação de novas propostas pedagógicas e institucionais tanto no Ensino Médio com a devida preparação para a nova fase, quanto no Ensino Superior, para que os alunos possam se ajustar à nova realidade e aproveitar as diversas possibilidades e perspectivas para seu futuro profissional. 
Ao observar o contexto dos calouros do curso de Psicologia do Estado do Rio de Janeiro, verificou-se que a adaptação mais satisfatória esteve associada a maiores níveis de Expectativas Acadêmicas, Educação à Carreira e Habilidades Sociais. Podendo-se inferir, assim, que os estudantes que ingressam nesta modalidade de Curso Superior e alcançam determinado sucesso inicial são os jovens que idealizaram sua entrada no Ensino Superior, receberam apoio educacional para a sua escolha e apresentam maior capacidade de relacionamentos interpessoais. Estes dados apontam para duas realidades importantes para a Educação Básica brasileira, uma preparação adequada para o processo de escolha profissional e a formação para a competência social, aspectos que ainda não são tão expressivos no modelo educacional do Brasil.

Algumas limitações podem ser apresentadas como a própria característica específica da amostra. É importante ressaltar, portanto, que novas pesquisas com estudantes de diversos cursos, períodos universitários e de diferentes regiões, podem apontar a forma como as variáveis Expectativas Acadêmicas, Habilidades Sociais, Maturidade para a Escolha Profissional e a Educação à Carreira estão relacionadas à Adaptação Acadêmica nos diversos contextos estudantis. 


\section{Referências}

Almeida, L. S., Soares, A. P., \& Ferreira, J. A. G. (2002). Questionário de Vivências Acadêmicas (QVAr): Avaliação do ajustamento dos estudantes universitários. Avaliação Psicológica, 1(2), 81-93.

Balbinotti, M. A. A., \& Tétreau, B. (2006). Questionário de educação à carreira: propriedades psicométricas da versão brasileira e comparação transcultural. Revista Brasileira de Orientação Profissional, 7(2), 49-66.

Blustein, D. L. (2011). A relational theory of working. Journal of Vocational Behavior, 79(1), 1-17. Doi:10.1016/j.jvb.2010.10.004

Carvalho, T. O., \& Marinho-Araújo, C. M. (2010). Psicologia Escolar e Orientação Profissional: Fortalecendo as convergências. Revista Brasileira de Orientação Profissional, 11(2), 219-228.

Colombo, G, \& Pratti, L, E. (2014). Maturidade para Escolha Profissional, Habilidades Sociais e Inserção no Mercado de Trabalho. Revista Brasileira de Orientação Profissional, 15(2), 201-212.

Del Prette, A., \& Del Prette, Z. A. P. (2003). No contexto da travessia para o ambiente de trabalho: Treinamento de habilidades sociais com universitários. Estudos de Psicologia, 8(3), 413-420.

Del Prette, Z. A. P, \& Del Prette, A. (2013). Psicologia das habilidades sociais na infância: Teoria e Prática. Petrópolis, RJ: Vozes.

Del Prette, Z. A. P., \& Del Prette, A. (2001). Inventário de Habilidades Sociais (IHS-Del-Prette): Manual de aplicação, apuração e interpretação. São Paulo: Casa do Psicólogo.

Del Prette, Z. A. P., \& Del Prette, A. (2003). Desenvolvimento interpessoal: Uma questão pendente no ensino universitário. In E. Mercuri e S. A. J. Polydoro, (Org.). Estudante universitário: Características e experiências de formação (pp. 105-128). Taubaté: Cabral Editora e Livraria Universitária.

Faleiros, N. P., \& Lehman, P. Y. (2016). Desafios na implantação da educação para a carreira no contexto escolar brasileiro. Revista Brasileira de Orientação Profissional, 17(2), 233-243.

Fernandes, E. P., \& Almeida, L. S. (2005). Expectativas e vivências acadêmicas: Impacto no rendimento acadêmico dos alunos do primeiro ano. Psychologica, 40(3), 267-278.

Figueiredo, A. C., Filho, A. Del M., A., \& Santos, L. M. (2015). Carreira Tradicional ou Moderna? Um estudo com alunos da rede municipal de ensino de São José dos Campos-SP. Revista de Empreendedorismo e Gestão de Pequenas Empresas, 5(1), 163-191.

Gamboa, V. M., \& Paixão, M. P. (2014). A qualidade da experiência de estagio e o desenvolvimento vocacional de estudantes dos cursos tecnológicos. Psicologia: Reflexão e Crítica, 27(2), 377-387. Doi:10.1590/1678-7153.201427219

Gomes, G., \& Soares, A. B. (2013). Inteligência, habilidades sociais e expectativas acadêmicas no desempenho de estudantes universitários. Psicologia: Reflexão e Crítica, 26(4), 780-789.

Granado, J. I. F., Santos, A. A. A., Almeida, L. S., Soares, A. P., \& Guisande, M. A. (2005). Integração académica de estudantes universitários: Contributos para a adaptação e validação do QVA-r no Brasil. Psicologia e Educação, 4(2), 31-41

Guichard, J. (2001). A century of career education: Review and perspectives. International Journal for Educational and Vocational Guidance, 1(3), 155-176.

Junqueira, M. L., \& Melo-Silva, L. L. (2014). Maturidade Para a Escolha de Carreira: Estudo com Adolescentes de um Serviço-Escola. Revista Brasileira de Orientação Profissional, 15(2), $187-$ 199.

Lisboa, F. S., \& Barbosa, A. J. G. (2009). Formação em Psicologia no Brasil: um perfil dos cursos de graduação. Psicologia: Ciência e Profissão, 29(4), 718-737. https://dx.doi.org/10.1590/S141498932009000400006

Marinho-Araújo C. M., Fleith, D. S., Almeida, L. S., Bisinoto, C., \& Rabelo, M. L. (2015). Adaptação da Escala Expectativas Acadêmicas de Estudantes Ingressantes na Educação Superior. Avaliação Psicológica, 14(1), 133-141.

Moreno, P. F., \& Soares, A. B. (2014). O que vai acontecer quando eu estiver na universidade? Expectativas de jovens estudantes brasileiros. Aletheia, 45, 114-127.

ARTICLES $\mid 442$ 
Nadelson, L. S., Semmelroth, C., Martinez, G., Featherstone, M., Fuhriman, C. A., \&Sell, A. (2013). Why did they come here? The influences and expectations of first-year student's college experience. Higher Education Studies, 3(1), 50-62.

Neiva, K. M. C. (2003). A maturidade para a escolha profissional: uma comparação entre alunos do ensino médio. Revista Brasileira de Orientação Profissional, 4(1-2), 97-103.

Neiva, K. M. C. (2014). Escala de Maturidade para a Escolha Profissional (EMEP). São Paulo: Vetor.

Neiva, K. M. C., Silva, M. B., Miranda, V. R., \& Esteves, C. (2005). Um estudo sobre a maturidade para a escolha profissional de alunos do Ensino Médio. Revista Brasileira de Orientação Profissional, $6(1), 1-14$.

Nelson, K. J., Quinn, C., Marrington, A., \& Clarke, J. A. (2012). Good practice for enhancing the engagement and success of commencing students. Higher Education, 63, 83-96.

Oliveira, C. T., Santos, A. S., \& Dias, A. C. G. (2016). Expectativas de universitários sobre a universidade: sugestões para facilitar a adaptação acadêmica. Revista Brasileira de Orientação Profissional, 17(1), 43-53.

Oliveira, I. M., Taveira, M. C., \& Neves, L. F. (2014). Sensibilizar professores para o desenvolvimento de carreira dos alunos: Relato de uma experiência. Psicologia: Ciência e Profissão, 34(2), 512-523.

Pellegrini, C. F. S., Calais, S. L., \& Salgado, M. H. (2012). Habilidades sociais e administração de tempo no manejo do estresse. Arquivos Brasileiros de Psicologia, 64(3), 110-129.

Pinho, A. P. M., Dourado, L. C. D. C., Aurélio, R. M., \& Bastos, A. V. B. (2015). A transição do ensino médio para a universidade: Um estudo qualitativo sobre os fatores que influenciam este processo e suas possíveis consequências comportamentais. Revista de Psicologia, 6(1), 33-47.

Sarriera, J. C., Paradiso, A. C., Schütz, F. F., \& Howes, G. P. (2012). Estudo comparativo da integração ao contexto universitário entre estudantes de diferentes instituições. Revista Brasileira de Orientação Profissional, 13(2), 163-172

Soares, A. B., \& Del Prette, Z. A. P. (2015). Habilidades sociais e adaptação à universidade: Convergências e divergências dos construtos. Análise Psicológica, 33(2), 139-151.

Soares, A. B., Francischetto, V., Dutra, B. M., Miranda, J. M., Nogueira, C. C. C., Leme, V. R., Araújo, A. M., \& Almeida, L. S. (2014). O impacto das expectativas na adaptação acadêmica dos estudantes no Ensino Superior. Psico-USF, 19(1), 49-60.

Soares, A. B., Poubel, L. N., \& Mello, T. V. S. (2009). Habilidades sociais e adaptação acadêmica: Um estudo comparativo em instituições de ensino público e privado. Aletheia, (29), 27-42.

Soares, A. B., Seabra, A. M. R., \& Gomes, G. (2014). Inteligência, autoeficácia e habilidades sociais em estudantes universitários. Revista Brasileira de Orientação Profissional, 15(1), 85-94.

Soares, A. P., Almeida, L. D. S., Diniz, A. A. P. M., \& Guisande, M. A. (2006). Modelo Multidimensional de Ajustamento de jovens ao contexto Universitário (MMAU): Estudo com estudantes de ciências e tecnologias versus ciências sociais e humanas. Análise Psicológica, 1(24),15-27.

Sparta, M., \& Gomes, W. B. (2005). Importância atribuída ao ingresso na educação superior por alunos do ensino médio. Revista Brasileira de Orientação Profissional, 6(2), 45-53.

Teixeira, M. A. P., Dias, A. C. G., Wottrich, S. H., \& Oliveira, A. M. (2008). Adaptação à universidade em jovens calouros. Psicologia Escolar e Educacional, 12(1), 185-202.

Teixeira, M. O., \& Calado, I. (2010). Avaliação de um programa de educação para a carreira: um projeto de natureza exploratória. Revista Brasileira de Orientação Profissional, 11(2), 213-218.

Ulusoy, F. M., \& Onen, A. S. (2014). The effects of the professional maturity levels of secondary school students on their academic motivations. Procedia Social and Behavioral Sciences, 143, 1153 1157.

Uvaldo, M. C. C., Garcia, M. L. D., Munhoz, I. M. S., \& Teixeira, M. O. (2012). Síntese das discussões e propostas do grupo de trabalho: Interfaces entre a orientação profissional, educação e psicologia escolar. Revista Brasileira de Orientação Profissional, 13(1), 125-128. 